\title{
COVID-19 Pneumonia Complicated by Acute Pulmonary Embolism
}

\author{
MAHMED $^{\mathrm{a}}$, M SULTANA $^{\mathrm{b}}$
}

(J Bangladesh Coll Phys Surg 2020; 38: 82-83)

DOI: https://doi.org/10.3329/jbcps.v38i0.47348

Two cases from Bangladesh Specialized Hospital (BSH), presenting with fever, cough, and dyspnea, diagnosed as COVID-19 pneumonia, confirmed with Reverse Transcription-Polymerase Chain Reaction (RT-PCR) presenting with typical findings at HRCT Chest. ${ }^{1}$ these cases evolved with respiratory deterioration and elevated serum D-dimer level. ${ }^{2}$ Figure 1 illustrates the case of a 64-year- old male got admitted to the hospital on day 7 of onset of symptoms; unenhanced chest CT on day 14 from the onset of fever showed bilateral peripheral ground-glass opacities (Fig 1, A). CT pulmonary angiography performed on day 7 of admission helped to diagnose acute pulmonary embolism (Fig 1, B). Figure
2 depicts the case of a 55-year-old female got admitted to the hospital on day 5 of onset of symptoms; unenhanced chest CT on day 20 of admission showed bilateral ground-glass opacities and consolidation in a peripheral distribution (Fig 2, A). CT pulmonary angiography 20 days after admission confirmed acute pulmonary embolism (Fig 2, B). Pulmonary embolism and deep venous thrombosis (DVT) have been reported to occur in other viral pneumonias, but not as frequently as in COVID-19 patients. ${ }^{3}$ Patients with COVID-19 are presenting mostly as pneumonia, it is important to address possibility of venous thromboembolism in these cases. ${ }^{4}$ In this scenario, respiratory deterioration with

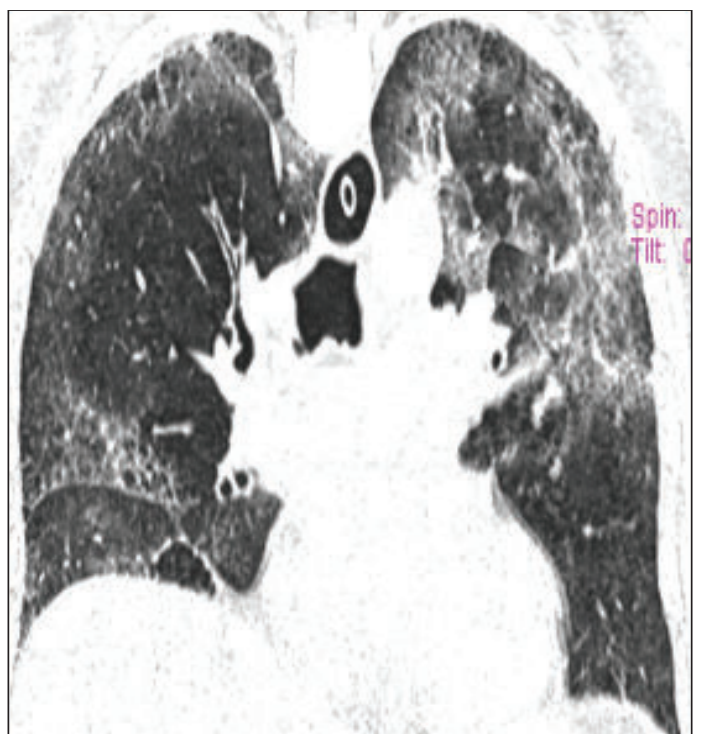

(a)

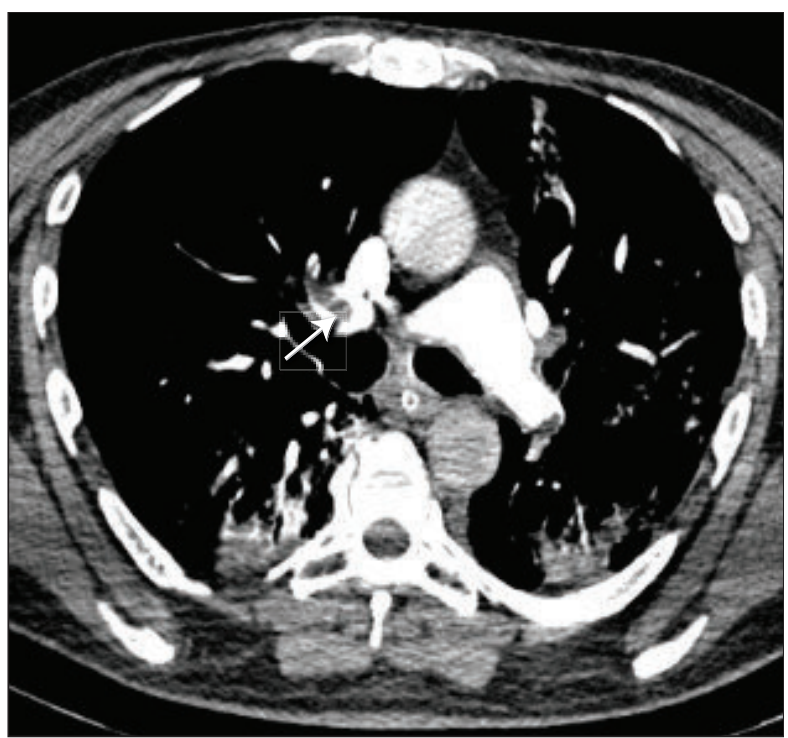

(b)

Fig.-1: Images in a 64-year-old male with COVID-19 pneumonia. A coronal reconstruction of unenhanced HRCT scan obtained on day 14 after the onset of symptoms shows (a) diffuse ground glass density with crazy paving pattern and obvious peripheral prominence. (b) CT pulmonary angiography demonstrates pulmonary embolism representing as filling defects in RPA (arrow) and the upper lobar branch, left interlobar artery.

a. Consultant, Medicine \& ICU, Bangladesh Specialized Hospital, Dhaka

b. Consultant, Radiology \& Imaging, Bangladesh Specialized Hospital, Dhaka

Address of Correspondence: M Ahmed, Consultant, Medicine \& ICU, Bangladesh Specialized Hospital, Dhaka, E-mail: m77ahmed@gmail.com 


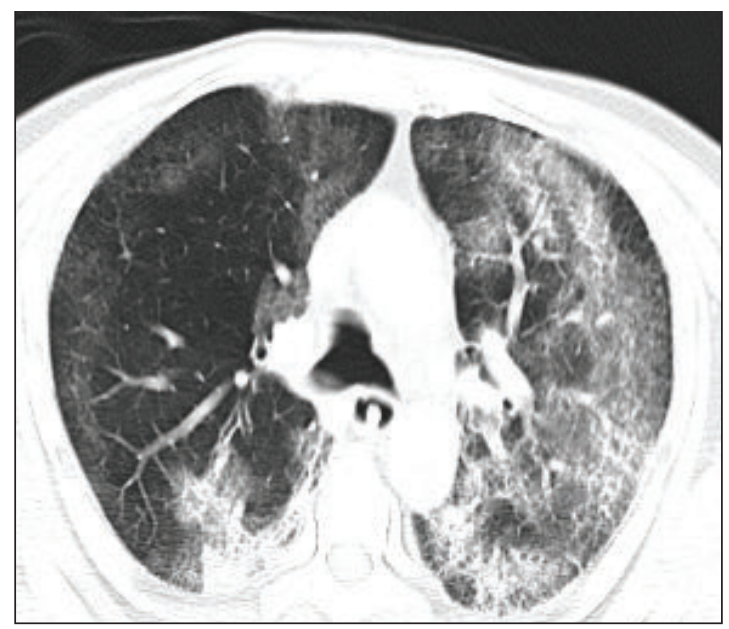

(a)

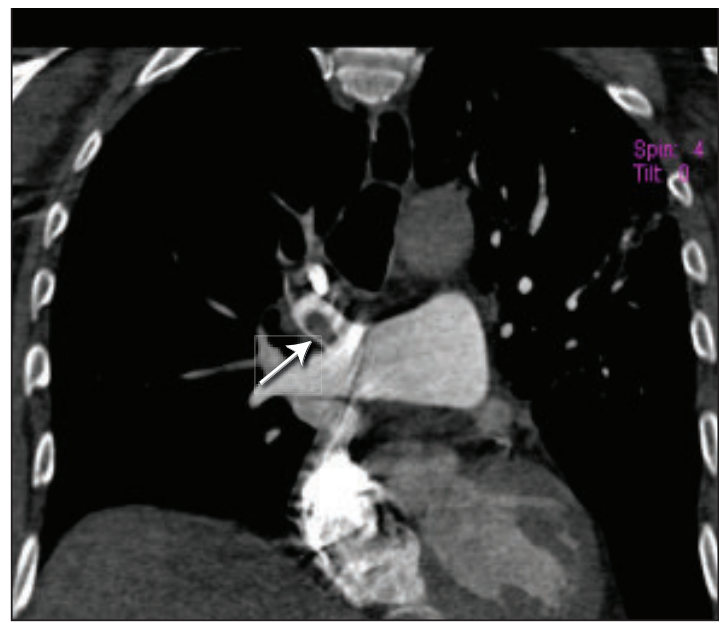

(b)

Fig.-2: Images in a 55-year-old female with COVID-19 pneumonia. A, Axial HRCT scan obtained on day 25 days after the onset of symptoms shows bilateral areas of peripheral ground-glass opacities, associated with crazy paving and peripheral consolidation suggesting infarct. B, CT pulmonary angiography demonstrates filling defect in RPA (arrow) and right upper lobar branch.

other clinical evidence of venous thrombosis should raise the suspicion of pulmonary embolism.

\section{Acknowledgments:}

We thank all colleagues for helping us during the current study. We are also grateful to the members of the healthcare workers (HCWs) for their selfless and heroic dedication in this pandemic, despite the potential threat to their own lives and the lives of their families.

Disclosures of Conflicts of Interest: A.M. disclosed no relevant relationships.

\section{References:}

1. Lei J, Li J, Li X. CT imaging of the 2019 novel coronavirus (2019-nCoV) pneumonia. Radiology 2020 https://doi.org/ 10.1148/radiol.2020200236.

2. Jolobe OM. Elevated D-dimer levels signify overlap between community-acquired pneumonia and pulmonary embolism.. Eur J Intern Med. 2013 Mar;24(2):e18. DOI: 10.1016/ j.ejim.2012.07.008. Epub 2012 Aug 21.

3. F Bompard. Pulmonary embolism in patients with Covid19 pneumonia. European Respiratory. Journal 2020; DOI: 10.1183/13993003.01365-2020

4. Charuhas Deshpande. Thromboembolic Findings in COVID19 Autopsies: Pulmonary Thrombosis or Embolism? Annals of Intern Med. 2020 May; https://doi.org/10.7326/M20-3255 